August 18, 1996

DUKE-TH-96-124

LBNL-39156, UCB-PTH-96/33

NSF-ITP-96-65

WIS-96/34/July-PH

hep-th/9608116

\title{
Supersymmetric Cycles in Exceptional Holonomy Manifolds and Calabi-Yau 4-Folds
}

\author{
Katrin Becker ${ }^{1}$, Melanie Becker ${ }^{2}$, David R. Morrison ${ }^{3}$, Hirosi Ooguri ${ }^{4,5}$, \\ Yaron $\mathrm{Oz}^{6}$, and Zheng $\mathrm{Yin}^{4,5}$ \\ 1 Institute for Theoretical Physics, University of California \\ Santa Barbara, CA 93106-4030, USA \\ 2 Department of Physics, University of California, Santa Barbara, CA 93106-9530, USA \\ ${ }^{3}$ Department of Mathematics, Duke University, Box 90320, Durham, NC 27708, USA \\ ${ }^{4}$ Department of Physics, University of California, Berkeley, CA 94720, USA \\ 5 Theoretical Physics Group, Ernest Orlando Lawrence Berkeley National Laboratory \\ University of California, Berkeley, CA 94720, USA \\ ${ }^{6}$ Department of Particle Physics, Weizmann Institute of Science 76100 Rehovot Israel
}

\begin{abstract}
We derive in the SCFT and low energy effective action frameworks the necessary and sufficient conditions for supersymmetric cycles in exceptional holonomy manifolds and Calabi-Yau 4-folds. We show that the Cayley cycles in $\operatorname{Spin}(7)$ holonomy eight-manifolds and the associative and coassociative cycles in $G_{2}$ holonomy seven-manifolds preserve half of the space-time supersymmetry. We find that while the holomorphic and special Lagrangian cycles in Calabi-Yau 4-folds preserve half of the space-time supersymmetry, the Cayley submanifolds are novel as they preserve only one quarter of it. We present some simple examples. Finally, we discuss the implications of these supersymmetric cycles on mirror symmetry in higher dimensions.
\end{abstract}




\section{Introduction}

A supersymmetric cycle is characterized by the property that the worldvolume theory of a brane wrapping around it is supersymmetric. The conditions for supersymmetric cycles in Calabi-Yau 3-folds have been analyzed using the low energy effective actions for branes $[1,2]$, where two types of conditions have been found. The first type corresponds to even-dimensional cycles being complex (holomorphic) submanifolds, i.e., having $\frac{1}{p !} k^{p}$ as their volume form, where $k$ denotes the Kähler form. The second type corresponds to middle-dimensional cycles being special Lagrangian, i.e., Lagrangian submanifolds having $\operatorname{Re}(\Omega)$ as their volume form where $\Omega$ corresponds to the nowhere vanishing holomorphic $(n, 0)$ form on the Calabi-Yau $n$-fold. The special Lagrangian and complex cycles in Calabi-Yau 3-folds and 4-folds have been shown in [3] to arise from the large volume limit of $N=2$ SCFT boundary conditions of $A$ and $B$ types respectively. Both types break half of the space-time supersymmetry.

Our aim is to study supersymmetric cycles of exceptional type that are not complex or special Lagrangian submanifolds, which exist in $\operatorname{Spin}(7), S U(4)$ and $G_{2}$ holonomy manifolds. For that it will be useful to introduce the concept of calibration [4] which is the appropriate framework to study supersymmetric cycles. A calibration is a closed $p$ form $\varphi$ on a Riemannian manifold of dimension $n$, such that its restriction to each tangent $p$-plane of $M$ is less or equal to the volume of the plane. Submanifolds for which there is equality are said to be calibrated by $\varphi$. A calibrated submanifold has the least volume in its homology class. In fact, this property of a calibration provides a natural geometric interpretation of the Bogomolnyi bound for D-branes wrapped about such submanifolds, with the calibrated submanifolds corresponding to the BPS states which saturate the bound. Complex and special Lagrangian submanifolds are calibrated by $\frac{1}{p !} k^{p}$ and $R e(\Omega)$ respectively. In addition to these calibrations there exist exceptional ones [4]. The Cayley calibration is a self-dual 4-form on eight-dimensional manifolds with holonomy contained in $\operatorname{Spin}(7)$. The associative calibration is a 3 -form on seven dimensional manifolds with holonomy contained in $G_{2}$, and the coassociative calibration is its Hodge dual.

In this paper we will analyze the supersymmetric cycles associated with these exceptional calibrations using the SCFT framework and the low energy effective action approach. In section 2 we will consider the Cayley calibration in $\operatorname{Spin}(7)$. In section 3 we consider $S U(4)$ holonomy eight-manifolds and the associative and coassociative calibrations in $G_{2}$ holonomy seven-dimensional manifolds are discussed in section 4 . We will construct the SCFT boundary conditions which in the large volume limit are associated with these cycles. We will find that the Cayley 4-cycle in $S U(4)$ holonomy Calabi-Yau 
4 -fold is novel as it preserves only one quarter of space-time supersymmetry, while all the others preserve as usual half of the supersymmetry. Using the supersymmetry transformations of the low energy effective action for branes compactified on the Calabi-Yau 4 -fold we derive the necessary and sufficient conditions for supersymmetric cycles. As expected, these conditions will coincide with the large volume limit of the SCFT boundary conditions. We present some simple examples of supersymmetric cycles in Calabi-Yau 4 -folds. In section 5 we discuss the implications of these supersymmetric cycles on mirror symmetry in higher dimensions.

\section{$2 \operatorname{Spin}(7)$ holonomy}

Let $M$ be an eight-manifold. A $\operatorname{Spin}(7)$ structure on $M$ is given by a closed self-dual $\operatorname{Spin}(7)$ invariant 4 -form $\Phi$. This defines a metric $g$ with holonomy group $\operatorname{Hol}(g) \subset$ $\operatorname{Spin}(7)$. Such a metric is Ricci-flat. Compact $\operatorname{Spin}(7)$ holonomy manifolds have been constructed in [5] by resolving the singularities of $T^{8} / \Gamma$ orbifolds. Here $T^{8}$ is equipped with a flat $\operatorname{Spin}(7)$ structure and $\Gamma$ is a finite group of isometries of $T^{8}$ preserving that structure. On a $\operatorname{Spin}(7)$ holonomy manifold there exists one covariantly constant spinor, which will provide us, upon compactification, with one space-time supersymmetry.

The 4-form $\Phi$ can be used as a calibration called the Cayley calibration. The calibration in general is related to the covariantly constant spinor via squaring [6] which basically means that the calibration form can be constructed from an appropriate product of two spinors.

The extended symmetry algebra of sigma models on $\operatorname{Spin}(7)$ manifolds has been found in [7]. In addition to the stress momentum tensor $T$ and its superpartner $G$, it contains two operators $\tilde{X}$ and $\tilde{M}$ with spins 2 and $\frac{3}{2}$ respectively. The presence of the spin 2 operator $\tilde{X}$ may be understood along the following lines: Recall that corresponding to the covariantly constant spinor there exists a dimension $\frac{1}{2}$ Majorana-Weyl spectral flow operator $\Psi_{L}$ mapping the Neveu-Schwarz (NS) sector to the Ramond sector. It implies the existence of a dimension 2 operator $\tilde{X}$, which is the energy-momentum tensor for the $c=\frac{1}{2}$ Majorana-Weyl fermion (Ising model), mapping the NS to NS sectors. In the large volume limit of the manifold $M, \tilde{X}$ takes the form [7]

$$
\tilde{X}_{L}=\frac{1}{2} g_{\mu \nu} \psi_{L}^{\mu} \partial_{z} \psi_{L}^{\nu}+\Phi_{\mu \nu \rho \sigma} \psi_{L}^{\mu} \psi_{L}^{\nu} \psi_{L}^{\rho} \psi_{L}^{\sigma},
$$

with a similar formula for $\tilde{X}_{R}$. The $\psi$ 's in (2.1) are the left handed fermions in the sigmamodel. This $\tilde{X}$ and its superpartner $\tilde{M}$ together with $T$ and $G$ make a closed algebra, and we will refer to it as the Ising superconformal algebra (ISCA). 
Let us impose now the boundary conditions. In order to preserve the $N=1 \mathrm{SCA}$ we require

$$
T_{L}=T_{R}, \quad G_{L}= \pm G_{R}
$$

Also, we have to preserve a linear combination of the left and right spectral flow operators. The ISCA algebra implies that

$$
\tilde{X}_{L}=\tilde{X}_{R}, \quad \tilde{M}_{L}= \pm \tilde{M}_{R} .
$$

Thus, there is only one type of boundary condition in this case.

The conditions (2.2) are solved in the large volume limit by

$$
\partial X^{\mu}=R_{\nu}^{\mu} \bar{\partial} X^{\nu}, \quad \psi_{L}^{\mu}= \pm R_{\nu}^{\mu} \psi_{R}^{\nu}
$$

where

$$
g_{\mu \nu} R_{\rho}^{\mu} R_{\sigma}^{\nu}=g_{\rho \sigma} .
$$

Here $X^{\mu}$ and $\psi^{\mu}$ denote coordinates and vielbein one-forms on the manifold. The eigenvectors of $R$ with eigen-values $(-1)$ give the Dirichlet boundary condition and thus correspond to the directions normal to the D-brane. As noted above, in the large volume limit $\tilde{X}$ takes the form (2.1). Using (2.4),(2.5) and (2.1) we see that the condition (2.3) reads

$$
\Phi_{\mu \nu \alpha \beta} R_{\rho}^{\mu} R_{\sigma}^{\nu} R_{\gamma}^{\alpha} R_{\delta}^{\beta}=\Phi_{\rho \sigma \gamma \delta} .
$$

Remembering that $\Phi$ is self-dual we see that the geometrical content of (2.6) is that $\Phi$ is the volume form of the supersymmetric cycle. Thus it is a Cayley submanifold as expected.

Since the boundary condition corresponding to the Cayley submanifold preserves a linear combination of the spectral flow operators we see that the $(2,0)$ space-time supersymmetry of type IIB compactified on $\operatorname{Spin}(7)$ holonomy manifold is broken by a D-brane wrapping on a Cayley submanifold to $(1,0)$.

\section{$3 \quad S U(4)$ holonomy}

\subsection{SCFT framework}

A Calabi-Yau 4-fold with $S U(4)$ holonomy posses two covariantly constant spinors of the same chirality. Thus, there exist two corresponding spectral flow operators $\Psi_{L}$ and $\Psi_{L}^{*}$ of dimension $\frac{1}{2}$. Combined with $\Psi_{R}$ and $\Psi_{R}^{*}$ we have four spectral flow operators which 
means that type IIB string compactified on a Calabi-Yau 4-fold to $1+1$ dimensions has $(4,0)$ space-time supersymmetry.

As we noted, supersymmetric cycles of special Lagrangian and holomorphic types are associated with $A$ and $B$ types of boundary conditions respectively [3]. These boundary conditions preserve two linear combinations of the spectral flow operators $\left\{\Psi_{L}, \Psi_{L}^{*}, \Psi_{R}, \Psi_{R}^{*}\right\}$ which implies that wrapping D-branes on these cycles breaks half of the space-time supersymmetry. Thus, the $(4,0)$ space-time supersymmetry is broken down to $(2,0)$.

One can also define for Calabi-Yau 4-folds an $S^{1}$ family of Cayley calibrations by

$$
\Phi_{\theta}=\frac{1}{2} k^{2}+R e\left(e^{i \theta} \Omega\right)
$$

Since $k^{2}$ vanishes on special Lagrangian submanifolds, and $\operatorname{Re}\left(e^{i \theta} \Omega\right)$ vanishes on complex submanifolds, the calibration (3.1) includes the special Lagrangian and complex calibrations as special cases. However a general Cayley submanifold is neither special Lagrangian nor complex. Note also that it cannot be simultaneously special Lagrangian and complex, since the Kähler form vanishes on Lagrangian submanifolds. Indeed we expect a special Lagrangian cycle and a complex 4-cycle to intersect transversely (at points) in the 4-fold.

In this section we study the Cayley type supersymmetric cycle. We will show that the boundary condition associated with the Cayley submanifold preserves only one linear combination of the four spectral flow operators and thus only a quarter of the space-time supersymmetry.

In view of the previous section, we know that we have to preserve the spin 2 operator $\tilde{X}$ corresponding to the energy momentum tensor of the preserved spectral flow operator. In order to formulate the boundary condition we embed the ISCA algebra in the $N=2$ SCA as

$$
T=T_{N=2}, \quad G=G_{N=2}^{+}+G_{N=2}^{-}, \quad \tilde{X}=\frac{1}{2} J^{2}+R e\left(e^{i \theta} \Omega\right),
$$

with $\tilde{M}$ as the superpartner of $\tilde{X}$. In the large volume limit $\tilde{X}$ takes the form

$$
\tilde{X}_{L}=\frac{1}{2} g_{\mu \nu} \psi_{L}^{\mu} \partial_{z} \psi_{L}^{\nu}+\left(\frac{1}{2} k^{2}+R e\left(e^{i \theta} \Omega\right)\right)_{\mu \nu \rho \sigma} \psi_{L}^{\mu} \psi_{L}^{\nu} \psi_{L}^{\rho} \psi_{L}^{\sigma}
$$

where we used the large volume limit expressions $J_{L}=g_{\mu \nu} \psi_{L}^{\mu} \psi_{L}^{\nu}$ and $\Omega=\Omega_{\mu \nu \rho \sigma} \psi_{L}^{\mu} \psi_{L}^{\nu} \psi_{L}^{\rho} \psi_{L}^{\sigma}$. Equation (3.3) is expected since as noted in (2.1), $\tilde{X}$ consists of two parts: The energy momentum tensor for the fermions and the Cayley calibration form, and the latter is given in (3.1). Note that in fact (3.2) defines an $S^{1}$ family of embeddings as suggested by (3.1). 
Let us also verify that $\tilde{X}$ is indeed the energy-momentum tensor for the Ising model. One way to see that is to bosonize the $U(1)$ current $J=i \partial_{z} \phi$ and use $\Omega=e^{i \phi}$. Thus,

$$
\tilde{X}_{L}=\frac{1}{2}\left(\partial_{z} \phi\right)^{2}+\cos (\phi+\theta)
$$

Combining the two spectral flow operators as $e^{i(\phi+\theta)}=\Psi_{1}+i \Psi_{2}$ we see that $\tilde{X}_{L}=\Psi_{1} \partial_{z} \Psi_{1}$, namely $\tilde{X}_{L}$ is the energy-momentum tensor for the Majorana-Weyl spinor $\Psi_{1}$ with $c=\frac{1}{2}$.

The boundary condition that corresponds to a Cayley submanifold which is neither special Lagrangian nor complex is that of (2.2) and (2.3). Thus, as we discussed, we are only preserving the energy-momentum tensor for one linear combination of spectral flow operators and break the rest of the $N=2 \mathrm{SCA}$. This leaves us with one quarter of the supersymmetry. The $S^{1}$ family of Cayley calibrations corresponds to the choice of the preserved linear combination of the spectral flow operators.

Until now the only known way for D-branes to break more than half of the spacetime supersymmetry was to use a configuration of intersecting branes [9]. The Cayley submanifold provides the first and the only example of a supersymmetric cycle on which a single wrapped D-brane breaks three quarters of the space-time supersymmetry.

\subsection{Low energy effective action framework, I}

In this and the following subsections we will use the low effective action framework in order to derive the conditions for supersymmetric cycles in Calabi-Yau 4-folds. This will make the space-time interpretation of the previous results manifest. To derive the conditions for having a supersymmetric 4-cycle, we consider the 3-brane of the ten-dimensional type IIB theory which wraps a 4-cycle of the Calabi-Yau 4-fold. The 3-brane solution of the type IIB theory was discovered in [10] and its static gauge field content is described by an abelian $D=4, N=4$ vector multiplet [11]. However, the covariant 3-brane action with the local $\kappa$ symmetry has not been constructed so far, so that it is hard to make a rigorous analysis along the lines of [1].

Alternatively, one may take the point of view that the low energy effective action for the Euclidean D3-brane is the "twisted" $N=4$ Yang-Mills theory [12] and count the number of unbroken supersymmetries by studying how the twisting is realized on the Cayley submanifold. According to [2], the twisting structure can be understood from the behavior of the normal bundle of the submanifold. For special Lagrangian submanifolds,

\footnotetext{
${ }^{*}$ That the energy-momentum tensor of the Ising model is given by (3.4) was shown in [8].
} 
the $S U(4)$ global symmetry of $N=4$ decomposes as $(2,1) \oplus(1,2)$ under the Lorentz group $S U(2) \times S U(2)$, which leads to 2 unbroken supersymmetries*

For a Cayley submanifold in a manifold with $\operatorname{Spin}(7)$-holonomy, the normal bundle can be written in the form [13] $\mathbb{S}_{-} \otimes \mathcal{F}$, where $\mathcal{F}$ is a certain rank two vector bundle on the Cayley submanifold. As pointed out in [2], if the vector bundle $\mathcal{F}$ is trivial then the twisting is the one for which the global $S U(4)$ symmetry decomposes as $(1,2) \oplus(1,1) \oplus$ $(1,1)$ under the Lorentz group; this leaves 1 unbroken supersymmetry. It remains to verify that in our situation - a Cayley submanifold of a manifold with $S U(4)$ holonomy which is neither a complex nor a special Lagrangian submanifold - the bundle $\mathcal{F}$ is trivial.

The structure of the normal bundle of a Cayley submanifold in the $\operatorname{Spin}(7)$ holonomy case is analyzed in some detail by McLean [13]. The half-spin representations of Spin(8) are eight-dimensional; if we fix a spinor $\sigma$ in one of the representation spaces, its stabilizer is isomorphic to $\operatorname{Spin}(7)$. Projecting that copy of $\operatorname{Spin}(7)$ to the vector representation of $\operatorname{Spin}(8)$ produces the holonomy representation $\operatorname{Spin}(7) \rightarrow S O(8)$. If the actual holonomy is $S U(4) \cong \operatorname{Spin}(6)$, there will be an embedding of $\operatorname{Spin}(6)$ in $\operatorname{Spin}(7)$, determined by a second spinor $\sigma^{\prime}$ of which $\operatorname{Spin}(6)$ is the stabilizer (within $\operatorname{Spin}(7)$ ).

Given a Cayley 4-plane $\xi$, there are quaternionic structures on the 4-planes $\xi$ and $\xi^{\perp}$ such that the stabilizer $G_{\xi}$ of $\xi$ in $\operatorname{Spin}(7)$ can be written as

$$
G_{\xi}=\left(S p(1)_{L} \times S p(1)_{L}^{\perp} \times S p(1)_{R}^{\text {diag }}\right) /\{ \pm(1,1,1)\},
$$

where $S p(1)_{L}$ and $S p(1)_{R}$ are the two natural subgroups of $S O(\xi)$ given by the left and right actions of the unit quaternions, $S p(1)_{L}^{\perp}$ and $S p(1)_{R}^{\perp}$ are the corresponding subgroups of $S O\left(\xi^{\perp}\right)$, and $S p(1)_{R}^{\text {diag }}$ is the diagonal subgroup of $S p(1)_{R} \times S p(1)_{R}^{\perp}$.

In terms of the embedding $\operatorname{Spin}(7) \subset \operatorname{Spin}(8)$, the group $\operatorname{Sp}(1)_{L} \times S p(1)_{L}^{\perp}$ is the stabilizer of a 4-plane $\eta$ of spinors orthogonal to $\sigma$, and $S p(1)_{R}^{\text {diag }}$ is the intersection of the stabilizer of $\eta^{\perp}$ with $\operatorname{Spin}(7)$. If we choose an embedding $\operatorname{Spin}(6) \subset \operatorname{Spin}(7)$ corresponding to a spinor $\sigma^{\prime}$, then there are three possibilities for the intersection of $\operatorname{Spin}(6)$ with $G_{\xi}$ :

1. $\sigma^{\prime} \in \eta^{\perp}$, in which case $\operatorname{Spin}(6) \cap G_{\xi}=S p(1)_{L} \times S p(1)_{L}^{\perp} \times U(1)$ with $U(1) \subset S p(1)_{R}^{\text {diag }}$, and

2. $\sigma^{\prime} \in \eta$, in which case $\operatorname{Spin}(6) \cap G_{\xi}=S U(2) \times S p(1)_{R}^{\text {diag }}$ with $S U(2) \subset S p(1)_{L} \times$ $S p(1)_{L}^{\perp}$ conjugate to the diagonal embedding

${ }^{*}$ The case of complex submanifolds is a bit different, and does not fall into the classification given in [12] since the normal bundle is not trivial before twisting. Nevertheless, in this case too there are 2 covariantly constant spinors. 
3. $\sigma^{\prime}$ generic, in which case $\operatorname{Spin}(6) \cap G_{\xi}=S U(2) \times U(1)$ with $S U(2) \subset S p(1)_{L} \times S p(1)_{\frac{1}{L}}^{\frac{1}{2}}$ conjugate to the diagonal embedding and $U(1) \subset S p(1)_{R}^{\text {diag }}$.

In case 1, the orbit of $\xi$ under $\operatorname{Spin}(6)=\operatorname{SU}(4)$ takes the form $\operatorname{Spin}(6) /\left(\operatorname{Spin}(6) \cap G_{\xi}\right) \cong$ $S U(4) / S(U(2) \times U(2))$, from which it is clear that $\xi$ is a complex subspace of $\mathbb{R}^{8}=\mathbb{C}^{4}$. In case 2 , the orbit of $\xi$ takes the form $\operatorname{Spin}(6) /\left(\operatorname{Spin}(6) \cap G_{\xi}\right) \cong S U(4) / S O(4)$ which implies that $\xi$ is a special Lagrangian 4-plane. Finally, in case 3 the orbit of $\xi$ takes the form $\operatorname{Spin}(6) /\left(\operatorname{Spin}(6) \cap G_{\xi}\right) \cong S U(4) /(S U(2) \times U(1))$ and has dimension 11 (different from the previous cases), so $\xi$ must be a Cayley 4-plane which is neither a complex nor special Lagrangian subspace.

To make contact with the SCFT approach in section 3.1, we can count the number of supersymmetries preserved, or equivalently, the number of supersymmetries broken. Each of the latter would generate a goldstino, i.e. a fermion zero modes in in the low energy effective super Yang-Mills action. In the present cases, they correspond to covariantly constant spinors. Generically, there are no more covariantly constant spinors. Thus the number of unbroken spacetime supersymmetries is equal to that of covariantly constant spinors in the fermion bundles of the low energy action. Using the intersection of Spin(6) with $G_{\xi}$ given above, we find them to be 2, 2, and 3 for complex, special lagrangian, and Cayley submanifolds respectively. Since the total number of spacetime supersymmetries in $\mathrm{SU}(4)$ compactification of type II string theory is 4 , this reproduces the counting given in section 3.2.

Following [13], when the holonomy is $\operatorname{Spin}(7)$, the vector bundle $\mathcal{F}$ in the normal bundles to a Cayley submanifold is the rank two bundle naturally associated to the principal bundle $S p(1)_{L}^{\perp}$. (In McLean's $p, q, r$ notation, $p \in S p(1)_{L}, q \in S p(1)_{R}^{\text {diag }}$ and $r \in S p(1)_{L}^{\perp}$.) Nonetheless, one can show, along the same line of reasoning employed above, that it is supersymmetric even when $\bar{F}$ is nontrivial.

\subsection{Low energy effective action framework, II}

Although a covariant 3-brane action with the local $\kappa$-symmetry is not yet known, it is not difficult to guess what its symmetry structure should be if there is one. By making a reasonable assumption on the symmetry structure of the would-be covariant action, one may formally extend the analysis of [1] to the present case, and give another derivation of the results of the previous subsection. The conditions satisfied by a supersymmetric 4 -cycle are expressed in terms of a holomorphic $(4,0)$-form:

$$
\Omega=\frac{1}{4 !} \Omega_{a b c d}(X) d X^{a} \wedge d X^{b} \wedge d X^{c} \wedge d X^{d},
$$


and the Kähler form

$$
k_{a \bar{b}}=i g_{a \bar{b}} .
$$

Here $a, \bar{b}$ are holomorphic and anti-holomorphic indices and $X^{i}$ are coordinates on the Calabi-Yau.

As discussed in subsection 3.1, an eight-manifold with $S U(4)$ holonomy has two covariantly constant eight-dimensional Majorana-Weyl spinors $\epsilon_{1}$ and $\epsilon_{2}$, with the same chirality. Changing the chirality of both spinors would correspond to reversing the orientation. We can combine these real spinors into a complex spinor $\epsilon_{+}=\epsilon_{1}+i \epsilon_{2}$, whose normalization can be chosen as $\epsilon_{+}^{\dagger} \epsilon_{+}=1$. The Kähler form is expressed in terms of this spinor as:

$$
k_{a}^{b}=i \epsilon_{+}^{\dagger} \gamma_{a}^{b} \epsilon_{+} .
$$

In general, $\gamma_{m_{1} \ldots m_{n}}$ is the completely antisymmetrized product of $n$ eight-dimensional gamma matrices containing a factor $1 / n$ !. From (3.8) it can be easily seen that $\gamma_{a}$ acts as an annihilation operator

$$
\gamma_{a} \epsilon_{+}=\gamma_{\bar{a}} \epsilon_{-}=0,
$$

where $\epsilon_{-}=\left(\epsilon_{+}\right)^{*}$. The holomorphic 4 -form relates $\epsilon_{+}$and $\epsilon_{-}$:

$$
\gamma_{a b c d} \epsilon_{-}=\Omega_{a b c d} \epsilon_{+} .
$$

Using standard properties of gamma matrices, it can be shown that the following formulas hold

$$
\begin{aligned}
\gamma_{a \bar{b} \bar{c} \bar{d} \epsilon_{+}} & =-3 i k_{a[\bar{b}} \gamma_{\bar{c} \bar{d}]} \epsilon_{+}, \\
\gamma_{\bar{a} \bar{b} c d} \epsilon_{+} & =3 k_{[\bar{a} c} k_{\bar{b} d]} \epsilon_{+} .
\end{aligned}
$$

Similar equations involving the spinor $\epsilon_{-}$can be obtained after complex conjugation.

Although the covariant action for the 3-brane is yet to be constructed, it should be natural to assume, by extending the analysis in [1], that the 3-brane would preserve the supersymmetries generated by ten-dimensional spinors $\epsilon$ if they solve*

$$
P_{-} \epsilon=\frac{1}{2}\left(1-\frac{1}{4 !} \epsilon^{\mu \nu \rho \sigma} \partial_{\mu} X^{M} \partial_{\nu} X^{N} \partial_{\rho} X^{P} \partial_{\sigma} X^{Q} \Gamma_{M N P Q}\right) \epsilon=0 .
$$

In the covariant formulation, this would be a condition for the local $\kappa$-transformation to compensate for the global supersymmetry generated by $\epsilon$. Here $M, N=1, \ldots, 10$ are

${ }^{*}$ By comparing with the analysis of section 3.2 using the $N=4$ Yang-Mills theory, we note that, in the case of a special Lagrangian submanifold, we have to take into account both projection operators $P_{+}$ and $P_{-}$. 
ten-dimensional indices, $\mu, \nu=1, \ldots, 4$ are the worldbrane indices ${ }^{\dagger}, \Gamma_{M}$ are the tendimensional gamma matrices and $X^{M}$ is the bosonic part of the 3-brane configuration. In the above formula we have introduced the projection operator $P_{-}$which is hermitian and satisfies $P_{-}^{2}=P_{-}$.

Let us introduce the eight-dimensional spinor:

$$
\epsilon_{\theta}=e^{-i \theta / 2} \epsilon_{+}+e^{i \theta / 2} \epsilon_{-}
$$

The spinor $\epsilon$ can then be written in the form $\epsilon=\lambda \epsilon_{\theta}$, where $\lambda$ is a two-dimensional Majorana spinor. The simplest way to find solutions of the equation $P_{-} \epsilon_{\theta}=0$, is to derive a Bogomolnyi bound (which will be closely related to the calibration condition, as we have remarked earlier). This bound is saturated if and only if the 4-cycle is supersymmetric. This implies that the 3 -brane has minimized its volume. The bound can be derived from the inequality

$$
\int d^{4} \sigma \sqrt{h}\left(P_{-} \epsilon_{\theta}\right)^{\dagger} P_{-} \epsilon_{\theta} \geq 0
$$

In the above formula $P_{-}$is constructed from eight-dimensional gamma matrices and $h$ is the induced metric on the 3-brane. After a straightforward computation we obtain the result:

$$
V_{4} \geq \frac{1}{2} \int k \wedge k+\operatorname{Re}\left(e^{i \theta} \int \Omega\right)
$$

Comparing to the Cayley calibration (3.1) we see that the manifolds which saturate this bound correspond to the $S^{1}$ family of Cayley submanifolds, that we previously found. As we already pointed out, Lagrangian submanifolds and complex submanifolds are special cases of Cayley geometries. The complex submanifolds found herein coincide precisely with the expression found in [1] for the supersymmetric 3-brane wrapping a 4-cycle of a Calabi-Yau 3-fold.

\subsection{Examples}

The simplest examples of supersymmetric 4-cycles can be found in flat space*. Here:

$$
\begin{aligned}
\Omega & =d X^{1} \wedge d X^{2} \wedge d X^{3} \wedge d X^{4} \\
k & =d X^{1} \wedge d X^{\overline{1}}+\ldots+d X^{4} \wedge d X^{\overline{4}} .
\end{aligned}
$$

${ }^{\dagger}$ This notation is different than the one used in the previous sections where the distinction between worldbrane indices and Calabi-Yau indices was encoded in the eigen-vectors of the $R$ matrix.

${ }^{*}$ Here we will solve (3.15) pointwise. 
An example of a Lagrangian submanifold is the surface described by $X^{i}=X^{\bar{i}}$ for $i=1, \ldots, 4$. In that case equation (3.15) is saturated because the pullback of $\Omega$ satisfies

$$
\partial_{\alpha} X^{m} \partial_{\beta} X^{n} \partial_{\gamma} X^{p} \partial_{\delta} X^{q} \Omega_{m n p q}=\epsilon_{\alpha \beta \gamma \delta},
$$

while the pullback of $k$ vanishes.

A more complicated example, that is not in flat space, can be found as a 4-cycle in the sextic hypersurface

$$
\sum_{i=1}^{6}\left(X^{i}\right)^{6}=0 .
$$

in $C P^{5}$. This 4-cycle is the four-dimensional submanifold on which all the $X^{i}$ 's are real $[14,1]$.

An example of a complex submanifold is given by the surface described by $X_{3}=X_{4}=$ 0 . Here the pullback of $\Omega$ vanishes and the pullback of $k \wedge k$ is

$$
\partial_{\alpha} X^{m} \partial_{\beta} X^{n} \partial_{\gamma} X^{\bar{p}} \partial_{\delta} X^{\bar{q}} k_{m \bar{p}} k_{n \bar{q}}=2 \epsilon_{\alpha \beta \gamma \delta},
$$

so that (3.15) is saturated.

An example of a Cayley geometry, for which both the pullback of the holomorphic 4-form and the pullback of $k \wedge k$ are non-vanishing is described by $X^{2}=\sqrt{2} e^{i \varphi}\left(X^{1}+X^{\overline{1}}\right)$ and $X^{4}=\sqrt{2} e^{i \varphi}\left(X^{3}+X^{\overline{3}}\right)$, for every value of the angle $\varphi$. More generally, every Cayley plane that is neither special Lagrangian nor holomorphic will give an example of this type.

\section{$4 \quad G_{2}$ holonomy}

\subsection{SCFT framework}

Let $M$ be an seven-manifold. A $G_{2}$ structure on $M$ is given by a closed $G_{2}$ invariant 3 -form $\Phi$. This defines a metric $g$ with holonomy group $\operatorname{Hol}(g) \subset G_{2}$. Such a metric is Ricci-flat. Compact $G_{2}$ holonomy manifolds have been constructed in $[15,16]$ in analogy with the $\operatorname{Spin}(7)$ holonomy case by resolving the singularities of $T^{7} / \Gamma$ orbifolds. Here $T^{7}$ is equipped with a flat $G_{2}$ structure and $\Gamma$ is a finite group of isometries of $T^{7}$ preserving that structure. On a $G_{2}$ holonomy manifold there exists one covariantly constant spinor. The 3 -form $\Phi$ and its Hodge dual 4 -form ${ }^{*} \Phi$ define the associative and coassociative calibrations respectively.

The extended symmetry algebra of sigma models on $G_{2}$ manifolds has been constructed in [7]. In addition to the stress tensor $T$ and its superpartner $G$, it contains the super-

partners $(K, \Phi)$ with spins $\left(2, \frac{3}{2}\right)$ and $(X, M)$ with spins $\left(2, \frac{5}{2}\right)$. In the large volume limit, 
$\Phi$ corresponds to the associative calibration 3 -form and $X$ is the sum of the coassociative calibration 4 -form ${ }^{*} \Phi$ and the stress tensors for seven Majorana-Weyl fermions. In analogy with the $\operatorname{Spin}(7)$ holonomy case where we viewed $\tilde{X}$ as the stress tensor corresponding to the dimension $\frac{1}{2}$ spectral flow operator, here we can view $X$ as the stress tensor corresponding to the dimension $\frac{7}{16}$ spectral flow operator which is the spin field of the $c=\frac{7}{10}$ tri-critical Ising model.

In addition to the $N=1$ boundary condition (2.2), the $G_{2}$ algebra implies the boundary conditions

$$
\begin{array}{ll}
\Phi_{L}=\Phi_{R}, & K_{L}= \pm K_{R}, \\
X_{L}=X_{R}, & M_{L}= \pm M_{R} .
\end{array}
$$

In the large volume limit we have

$$
\Phi_{L}=\Phi_{i j k} \psi_{L}^{i} \psi_{L}^{j} \psi_{L}^{k}, \quad X_{L}=\frac{1}{2} g_{i j} \psi_{L}^{i} \partial_{z} \psi_{L}^{j}+{ }^{*} \Phi_{i j k l} \psi_{L}^{i} \psi_{L}^{j} \psi_{L}^{k} \psi_{L}^{l}
$$

Thus the boundary conditions (4.1) take the form

$$
\Phi_{i j k} R_{l}^{i} R_{m}^{j} R_{n}^{k}=\Phi_{l m n}, \quad{ }^{*} \Phi_{i j k l} R_{m}^{i} R_{n}^{j} R_{o}^{k} R_{p}^{l}={ }^{*} \Phi_{m n o p}
$$

which geometrically mean that for the 3 -cycle $\Phi$ is the volume form while for a 4 -cycle ${ }^{*} \Phi$ is the volume form. These are the associative and coassociative calibrated submanifolds. Since the boundary conditions (4.1) impose one linear constraint on the stress tensor operator corresponding to the spectral flow we see that a brane wrapping on an associative or coassociative cycle preserves half of the space time supersymmetry. Thus, the $(2,0)$ space-time supersymmetry of a type IIB string compactified on a $G_{2}$ holonomy sevenmanifold is broken to $(1,0)$ by the brane.

\subsection{Low energy effective action framework}

Supersymmetric 3-cycles are defined as configurations for which we can find a sevendimensional spinor that satisfies

$$
P_{-} \epsilon=\frac{1}{2}\left(1-\frac{i}{3 !} \epsilon^{\mu \nu \rho} \partial_{\mu} X^{m} \partial_{\nu} X^{n} \partial_{\rho} X^{p} \Gamma_{m n p}\right) \epsilon=0 .
$$

This expression is evaluated using the 3 -form $\Phi$, which appears in the expression

$$
\gamma_{m n p} \epsilon=\Phi_{m n p} \epsilon
$$


From here we see that the only configuration that preserves supersymmetry satisfies that the pullback of the 3 -form is proportional to the volume element:

$$
\partial_{[\mu} X^{m} \partial_{\nu} X^{n} \partial_{\rho]} X^{p} \Phi_{m n p}=\epsilon_{\mu \nu \rho} .
$$

These are precisely the associative calibrations previously discussed. On a manifold with $G_{2}$ holonomy we can also have 4 -cycles in which ${ }^{*} \Phi$ pulls back to the volume element. These cycles satisfy

$$
P_{-} \epsilon=\frac{1}{2}\left(1-\frac{1}{4 !} \epsilon^{\mu \nu \rho \sigma} \partial_{\mu} X^{m} \partial_{\nu} X^{n} \partial_{\rho} X^{p} \partial_{\sigma} X^{q} \Gamma_{m n p q}\right) \epsilon=0,
$$

which is solved by the configuration

$$
\partial_{[\mu} X^{m} \partial_{\nu} X^{n} \partial_{\rho} X^{p} \partial_{\sigma]} X^{q *} \Phi_{m n p q}=\epsilon_{\mu \nu \rho \sigma} .
$$

Thus ${ }^{*} \Phi$ corresponds to the coassociative calibration. Both configurations break half of the supersymmetry.

\section{Mirror symmetry}

In the case of the 4-fold with $S U(4)$ holonomy, we may consider the effect of mirror symmetry which exchanges $G_{N=2}^{+}$and $G_{N=2}^{-}$for the right mover. As was shown in [3], mirror symmetry exchanges the $A$ and $B$ types of boundary conditions. Geometrically, mirror symmetry is realized on pairs of Calabi-Yau manifolds which define the same theory (but with opposite geometric identifications of $G_{N=2}^{ \pm}$). Thus, if $X$ and $Y$ are a pair of mirror manifolds, the special Lagrangian submanifolds of $X$ are mapped to the complex submanifolds of $Y$, and the complex submanifolds of $X$ are mapped to the special Lagrangian submanifolds of $Y$.

Mirror symmetry for 4-folds has several new features which distinguish it from the three-dimensional case [17]. Mirror symmetry is expected to map $H^{4}(X)=\oplus_{p} H^{p, 4-p}(X)$ to $\oplus_{p} H^{p, p}(Y)$ and $\oplus_{p} H^{p, p}(X)$ to $H^{4}(Y)=\oplus_{p} H^{p, 4-p}(Y)$; one of the new features is that $H^{2,2}(X)$ appears in both of these spaces. (These spaces were referred to as the "horizontal" and "vertical" cohomology in [17].)

The special Lagrangian submanifolds of $X$ define classes in $H^{4}(X)$ which lie in the socalled primitive cohomology, that is, they are classes which are orthogonal to the Kähler class. Since the classes of special Lagrangian submanifolds are also classes in integer cohomology, the natural space to consider for these manifolds is $H^{4}(X)_{\text {prim }} \cap H^{4}(X, \mathbb{Z})$. It is not clear how much of this space will actually be represented by special Lagrangian submanifolds. 
On the other hand, the complex submanifolds of $X$ define classes which have Hodge type $(p, p)$ and are also integer cohomology classes; the natural space to consider for them is $\bigoplus_{p} H^{p, p}(X) \cap H^{\text {even }}(X, \mathbb{Z})$. The celebrated "Hodge conjecture" in mathematics asserts that if we pass to $\mathbb{Q}$-coefficients instead of $\mathbb{Z}$-coefficients, then all classes in this space are represented by complex submanifolds; it is not known if this conjecture holds for Calabi-Yau 4-folds.

We are thus faced with the situation of having an unknown subspace of $H^{4}(X)_{\text {prim }} \cap$ $H^{4}(X, \mathbb{Z})$ represented by special Lagrangian submanifolds, and an unknown subspace of $\oplus_{p} H^{p, p}(X) \cap H^{\text {even }}(X, \mathbb{Z})$ represented by complex submanifolds. In fact, it is quite possible that the appropriate pieces of these subspaces fall short of filling out all of $H^{2,2}(X)$ (even though both will contribute subspaces of $H^{2,2}(X)$ ). Cayley submanifolds provide another potential source of cohomology classes which could help to fill out $H^{2,2}(X)$ : it may be that some of the classes which cannot be represented by either special Lagrangian or complex submanifolds will instead be represented by Cayley submanifolds.

Such a possibility meshes well with mirror symmetry: we observe that the mirror of a Cayley submanifold will be another Cayley submanifold. (This is because any D-brane on $X$ - which defines some type of boundary condition for open strings - should map to a Dbrane on $Y$.) If the first Cayley submanifold is neither special Lagrangian nor a complex submanifold, then since it preserves only $1 / 4$ of the supersymmetry, its mirror will have the same property. It would be interesting to find explicit examples of this phenomenon.

Finally, we would like to mention an implication for mirror symmetry in higher dimensions that becomes evident by considering the spectrum of BPS soliton states. Recently Strominger, Yau and Zaslow [18] showed that every Calabi-Yau 3-fold that has a mirror admits a supersymmetric $T^{3}$-fibration. The basic assumption of this argument is quantum mirror symmetry $[20,21,1,22]$, where the isomorphism between the type IIA theory compactified on a 3-fold $X$ and the IIB theory compactified on the mirror $Y$ of $X$ is extended to the non-perturbative BPS states in $D=4$. Since these BPS states are constructed as D-branes, the quantum mirror symmetry is actually a consequence of the classical mirror symmetry of the bulk CFT [3]. It is then natural to wonder if the previous argument can be extended to higher dimensional Calabi-Yau manifolds. Some precise mathematical aspects of this generalization have been recently considered in [19]. We consider the type IIA theory compactified on a large Calabi-Yau $n$-fold $X$ and its mirror $Y$. Quantum mirror symmetry implies that both theories are isomorphic. On the $X$ side there are 'BPS states' in $D=(10-2 n){ }^{*}$ which arise from the ten-dimensional 0-brane. These states

\footnotetext{
${ }^{*}$ Rigorously, the notion of a BPS state that carries electro-magnetic charge in $D \leq 2$ is not well defined
} 
arise from a supersymmetric $n$-brane wrapping a $n$-cycle in $Y$. This $n$-cycle corresponds to a special Lagrangian submanifold. This is because the 0-brane corresponds to B-type boundary conditions and by mirror symmetry these are transformed to the A-type boundary conditions that correspond to the special Lagrangian submanifold [3]. Extending the arguments of [18] to $n$-folds, we arrive at the conclusion that the $n$-cycles corresponding to special Lagrangian submanifolds are toroidal. This leads us to the conclusion that every Calabi-Yau $n$-fold that has a mirror admits a supersymmetric $T^{n}$-fibration. This suggests that the mirror symmetry for the $n$-fold is equivalent to a T-duality on the $T^{n}$-fibers.

\section{Acknowledgements}

We would like to thank R. Bryant, G. Moore, R. Plesser, J. Polchinski, A. Schwimmer, A. Strominger, C. Vafa, and Y. Vlassopoulos for useful discussions. D.R.M. and H.O. are grateful for the hospitality of the Aspen Center for Physics during the final stages of this project. The work of K.B. was supported by NSF grant PHY89-04035. The work of M.B. was supported by DOE grant DOE-91ER40618. The work of D.R.M. was supported in part by the National Science Foundation under grant DMS-9401447. The work of H.O. was supported in part by NSF grant PHY-951497 and DOE grant DE-AC03-76SF00098. Y.O. is partially supported by the Israel Science Foundation through the Center for the Physics of Basic Interactions. Z.Y. is supported by a Graduate Research Fellowship of the U.S. Department of Education.

due to the occurrence of infrared divergences. (We thank A. Strominger for pointing this out to us). The problem can be better formulated by translating the result of [18] into the CFT language. 


\section{References}

[1] K. Becker, M. Becker and A. Strominger, "Fivebranes, Membranes and Nonperturbative String Theory," hep-th 9507158, Nucl. Phys. B456 (1995) 130.

[2] M. Bershadsky, V. Sadov and C. Vafa, "D-Branes and Topological Field Theories," hep-th 9511222, Nucl. Phys. B463 (1996) 420.

[3] H. Ooguri, Y. Oz and Z. Yin, "D-branes on Calabi-Yau Spaces and Their Mirrors," hep-th 9606112, To appear in Nucl.Phys.B.

[4] R. Harvey and H. B. Lawson, "Calibrated Geometries," Acta. Math. 148 (1982) 47.

[5] D. Joyce, "Compact 8-Manifolds with Holonomy Spin(7)," Invent. Math. 123 (1996) 507.

[6] R. Harvey, "Spinors and Calibrations," Academic Press (1990).

[7] S. Shatashvili and C. Vafa, "Superstrings and Manifolds of Exceptional Holonomy," hep-th 9407025, Selec. Math. 1 (1995) 347.

[8] T. Eguchi and K. Higashijima, "Vertex Operators and Nonabelian Bosonization", Proceedings of the Niels Bohr Centennial Symposium, Copenhagen, Denmark, May 6-10, 1985.

[9] M. Berkooz, M. R. Douglas and R. G. Leigh, "Branes Intersecting at Angles," hep-th 9606139 and references therein.

[10] G. T. Horowitz and A. Strominger, "Black Strings and P-Branes", Nucl. Phys. B360 (1991) 197.

[11] M. J. Duff and J. X. Lu, "The Self-Dual Type IIB Superthreebrane", Phys. Lett. 273B (1991) 409.

[12] C. Vafa and E. Witten, "A Strong Coupling Test of S-Duality," Nucl. Phys. B431 (1994) 3.

[13] R. C. McLean, "Deformations of calibrated submanifolds," J. Differential Geom. , to appear, preprint available at http://www.math.duke.edu/preprints/1996.html.

[14] R. L. Bryant, "Minimal Lagrangian Submanifolds of Kähler-Einstein Manifolds", Differential Geometry and Differential Equations, Shanghai 1985, Lecture Notes in Math., vol. 1255, Springer-Verlag, 1985, p. 1.

[15] D. Joyce, "Compact Riemannian 7-Manifolds with Holonomy $G_{2}$ I," J. Diff. Geom. 43 (1996) 291.

[16] D. Joyce, "Compact Riemannian 7-Manifolds with Holonomy $G_{2}$ II," J. Diff. Geom. 43 (1996) 329.

[17] B. R. Greene, D. R. Morrison and M. R. Plesser, "Mirror Manifolds in Higher Dimension," Comm. Math. Phys. 173 (1995) 559. 
[18] A. Strominger, S.-T. Yau and E. Zaslow, "Mirror Symmetry is T-Duality", hep-th/9606040.

[19] D. R. Morrison, "The Geometry Underlying Mirror Symmetry", alg-geom/9608006.

[20] A. Strominger, "Massless Black Holes and Conifolds in String Theory", Nucl. Phys. B451 (1995) 96.

[21] P. S. Aspinwall and D. R. Morrison, "U-Duality and Integral structures", Phys. Lett. 141B (1995) 141.

[22] D. R. Morrison, "Mirror Symmetry and the Type II String", hep-th/9512016, Trieste Conference on S-Duality and Mirror Symmetry, Nucl. Phys. B Proc. Suppl. 46 (1996), 146. 